\title{
Visual Simulation of Node Localization for WSN Based on Vega Prime
}

\author{
Qinghui Wang, Lei Chang, Wenzhou Wang, Jiao Li \\ College of Information Engineering, Shenyang University of Chemical Technology, Shenyang, China \\ Email: cl19870311@163.com
}

\begin{abstract}
Visual simulation can simulate a variety of situations of actual scene in real-time through the establishment of the virtual scene similar to a real scene. This paper studies on the node localization system for WSN (Wireless Sensor Network). In order to be able to observe the specific information of the node location for the wireless sensor network visually, we can construct scene model and texture process by Creator, then use Vega Prime to develop the visual simulation of node localization for wireless sensor network in the VS2005 platform, and demonstrate the node localization for wireless sensor network in real time and realistically.
\end{abstract} Prime

Keywords-Visual simulation; Wireless Sensor Network; Vega

\section{INTRODUCTION}

Visual simulation technology can structure the 3D model of simulation object and reproduce the real environment based on the purpose of simulation and using computer graphics technology, information integrated technology and display technology to achieve very realistic simulation effect. It includes building simulation environment and simulation driving. Building simulation environment mainly includes: model design, scene structure, texture design, special effects design, etc. It requires structuring the realistic 3D model and making lifelike texture and special effects. The simulation driving mainly includes: scene driving, model transfer processing, distribution interaction, large terrain disposal, etc. It requires fast and realistic reappearance simulation environment and real-time response interactive operation, etc. Visual simulation technology is conducive to shorten the test and development cycle, improve test and development quality, save test and development funds. It has been widely used in the military, engineering, aerospace, medical, construction and many other fields [1]. In particular, it provides a great convenience for people exploring the movement rule of things which is not convenient to observe directly because of a variety of reasons in the macroscopic and microscopic world.

Today wireless sensor network (WSN) is a widely concerned hot research area. in the world, it is integrated MEMS (Micro-Electron Mechanical System), sensor acquisition technology, data processing technology, embedded technology, data transmission technology and wireless transfer technology, etc [2]. In the application of wireless sensor network, when the layout of nodes is completed, node location problem was called by a certain way to know precisely their own position problem. Because of the necessary demand of the application, in the past few years the problem of sensor node localization was paid a lot of attention to and has become a hot topic in the research field of wireless sensor network [3]. In wireless sensor network, the nodes positioning mechanism refers to the limited beacon nodes whose position are known to determine other nodes position, the nodes in the wireless sensor network set up localization mechanism of location related. Target monitoring and tracking, intelligent transportation, logistics management and many applications require sensor node to provide their own position information.

General node localization for wireless sensor network demo interface is based on a 2D graphic interface display, it displays mobile node in 2D interface without verisimilitude, solid. This paper introduced the working process and realization method that the visual simulation of node realtime localization for wireless sensor network. Through the powerful 3D rendering technique of Vega Prime and VS2005 (Visual Studio 2005) platform, it simulates the scene of node localization for wireless sensor network intuitively and vividly. The objects in the scene can be driven by moving of the location node in wireless sensor network. The investigator can observe the real-time interaction and demo of real environment and the simulation scene easily and directly.

\section{THE DEVELOPMENT SOFTWARE}

In order to achieve realistic effect in real-time visual simulation platform, it needs to set up 3D scene model beforehand. In the process of simulation, real-time rendering of the complex 3D scene model will be required. According to the characteristics of the visual simulation, modeling tools MultiGen Creator and visual simulation development tools Vega Prime provided by Paradigm company are undoubtedly leading modeling and simulation software platforms in the field of virtual reality industry.

\section{A. MultiGen Creator}

MultiGen Creator integrated many kinds of advanced features in the polygon modeling, terrain generation and so on It is different from the main characteristics of traditional 3D modeling software packages, and does not lie in its powerful polygon modeling function but in its original hierarchical data structure-OpenFlight (.flt) data structure used to describe 3D virtual scene [4]. OpenFlight that takes the node type of the hierarchical structure can quickly and easily edit, modify, and control the internal arbitrary elements of the scene directly, especially is suitable for realtime rendering operation of image generator. In addition, it 
provides such as the level of detail (LOD), the degree of freedom (DOF), and other advanced real-time function.

\section{B. Vega Prime}

Vega Prime is a high performance software environment and tools that used for real-time simulation and virtual reality application. The bottom of Vega Prime based on open graphics library OpenGL technology. Vega Prime configuration mainly includes: LynX graphical user interface, function call library based on $\mathrm{C}++$, and complete user documentation. The system architecture of Vega Prime as shown in Figure 1 [5].

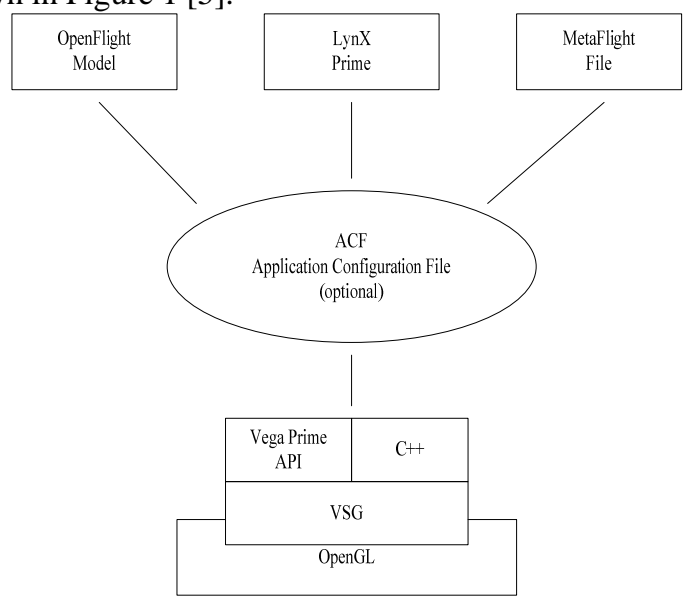

Figure 1. The system architecture of Vega Prime

Developing visual simulation system mainly includes three stages by Vega Prime: the preprocessing of visual mode, the configuration of visual model, the driver program design of visual simulation. The stage of visual model preprocessing mainly includes the static modeling of the scene terrain, such as the earth, the sea, forest, building and so on, also includes the complex entities modeling, such as aircraft cockpit, flight instrument, etc. The configuration of visual model sets position to all kinds of model imported, initializes the scene environment, and sets the special effects of collision, explosion and large scene application settings in the LynX Prime graphic interface. The simulation driving is the key stage of visual simulation; it completes the whole simulation processing. It calls the configured .acf file in the LynX, which receives the real-time simulation data that used to drive scene model movement which originate from backend database, and processes and controls the special effects in the scene, will provide real-time and fluid visual simulation results to users.

Vega Prime is the next generation products of Vega. Vega adopts the Performer software package of SGI Company and makes use of $\mathrm{C}$ interface. Vega Prime which adopts scene gallery of Vega Scene Graph (VSG) makes use of $\mathrm{C}++$ interface. The so-called scene gallery means that it allows users to express objects in the scene by using data structure, so that you can classify the related objects that shared some properties into group, and designate the whole group of common attributes in a place. Scene gallery can automatic management, truthfully draw the things scene required (such as LOD), and ignore the unnecessary details that slowing scene real-time display.

\section{VISUAL SIMULATION SYSTEM DESIGN}

\section{A. Modeling}

.The construction of 3D entity model is the foundation of $3 \mathrm{D}$ virtual scene. The modeling work is mainly divided into the experiment site modeling of the wireless sensor network and the driven object modeling.

By collecting model data, geometric modeling, model finishing and optimization, and then doing the texture mapping in the surface of the model that has been created, then the vivid and exquisite simulation model is formed It uses deleting redundancy surface, merger redundancy surface, the file format of conversion and other model optimization techniques.

MultiGen Creator contains a set of powerful tools which is used to construct a hierarchical visual database in the environment of what you see is what you get. The function of the tools mainly is to create, edit model and database, including dynamic reconfiguration database (once a database is built, and its hierarchical structure soon will be restructured, and the entire organization of the elements also be modified), the generation of dynamic instrument, the generation real-time terrain and so on. It can carefully edit individual element which is easily separated out. All sorts of color palettes can store the specific element, such as light and texture for repeated use.

The experiment site model created by creator has been optimized and texture processed is shown in Figure 2. A sphere is created as a driven object for node localization in wireless sensor network.

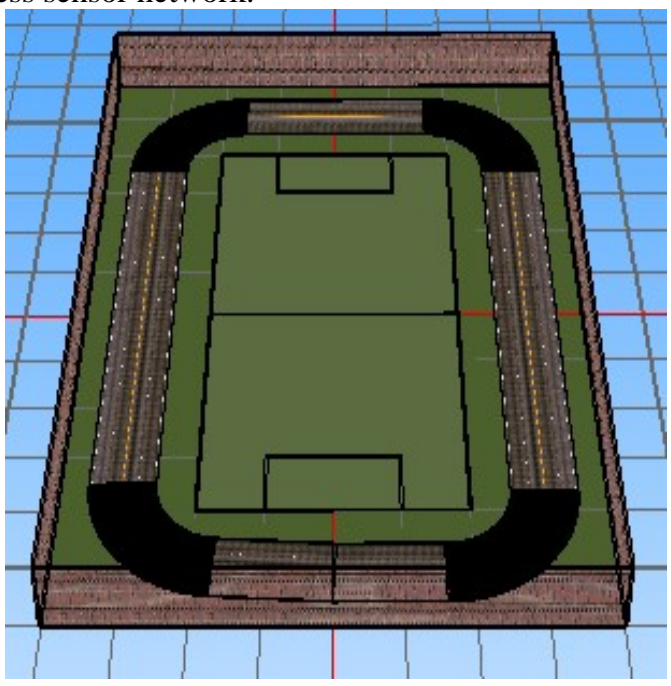

Figure 2. The experiment site model

\section{B. Visual simulation flow}

Using Vega Prime for visual simulation must first make use of Creator for scene modeling, and then using the LynX Prime of Vega Prime imports the model into Vega Prime, sets up the Application Configuration File (ACF) of scene, 
and saves the ACF; finally using Vega Prime develops the visual simulation for node localization in wireless sensor network in VS2005 platform. Figure 3 shows the Vega Prime system simulation flow.

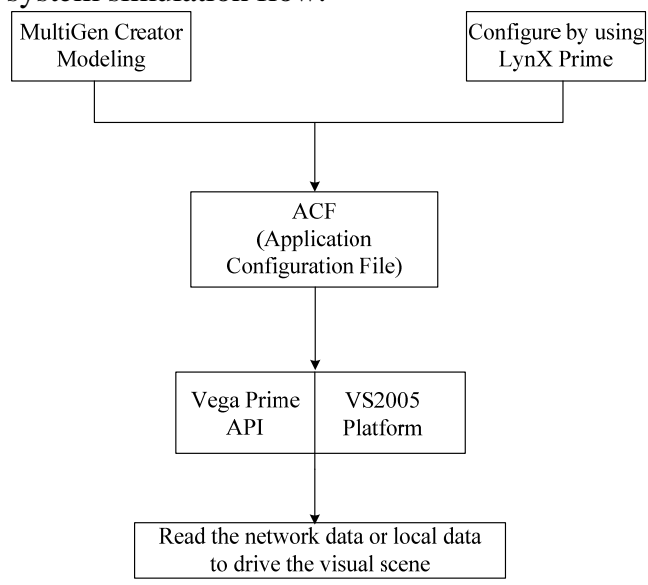

Figure 3. The Vega Prime system simulation flow

\section{The system of wireless sensor network}

The visual simulation of the wireless sensor network development system is the Germany Nanotron Company nanoLOC development kits, these development kits based on nanoLOC transceiver, with the characteristics of perfect design and the function of easy to use. They can be used for RTLS (Real Time Location System) and rapid development with position perception function system. The development kits as shown in Figure 4 shows:

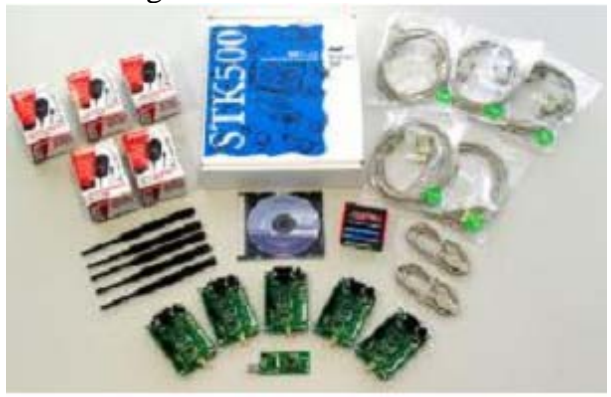

Figure 4. nanoLOC development kits

NanoLOC is to use CSS communication technology with high integration mixed signal chip. CSS (Chirp Spread Spectrum) works in ISM2.4 GHz, which used for battery power supply and medium speed applications, provides reliable transmission. Key Properties of CSS:

- High robustness: Due to the high Bandwidth $\times$ Time and their asynchronous nature, chirp pulses are very resistant against interferers.

- Multipath resistant: Due to the frequency spreading of chirp pulses, CSS is very immune against multipath fading; CSS can even take advantage of RF echoes.

- Long range: Due to high system gain, as well as noise, interference and fading resistance, CSS has exceptional range for a given transmit power and conditions.

- Low PHY latency: With CSS a wireless connection can be established very quickly because carrier frequency and data clock synchronizations are instantaneous.

- Location awareness: CSS systems have the inherent ability to determine the distance (range) between two stations.

So in this visual simulation system, the nanoLOC development kits were used.

\section{The system design of node localization for wireless sensor network and visual simulation}

General node localization for wireless sensor network demo interface is a visual plane coordinate system by using programming software to develop. It uses a dynamic point to display the position of the mobile node in the experiment, but can not demonstrate the actual scene intuitively and three-dimensional. However it can make a 3D visual simulation by using Vega Prime and VS2005. It used mobile node localization information of wireless sensor network to drive the movement of the object in 3D scene. Fig. 5 is the visual simulation system of node localization for wireless sensor network.

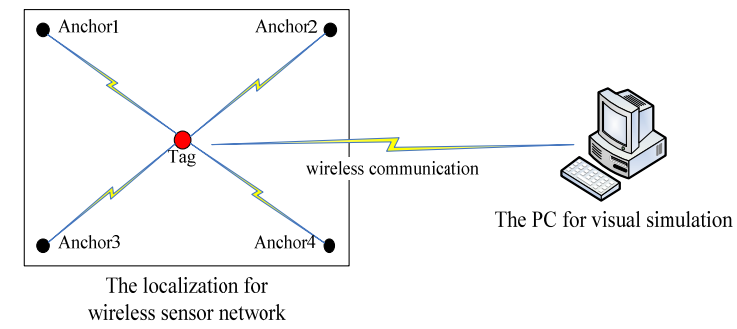

Figure 5. The visual simulation system of node localization for wireless sensor network

In Figure 5, Anchor 1-Anchor 4 which are fixed with the nodes of wireless sensor network system constitute a closed area, and set the node a coordinate, Tag is mobile node. In wireless sensor network through measured the distance between the fixed nodes and the mobile node, then the Tag node coordinate is calculated by localization algorithm. The Tag node sends the obtained coordinate information to the computer with the visual simulation function. Visual simulation computer makes use of the coordinate information to drive the movement of the sphere in $3 \mathrm{D}$ scene.

\section{VISUAL SIMULATION RESULTS}

The experiment of the wireless sensor network localization system was nanoLOC development kits, Figure 6 shows the 2D demo interface of the wireless sensor network localization, and the black spot stands for the mobile node. Figure 7 shows the visual simulation demonstration effect which designed by this paper. 


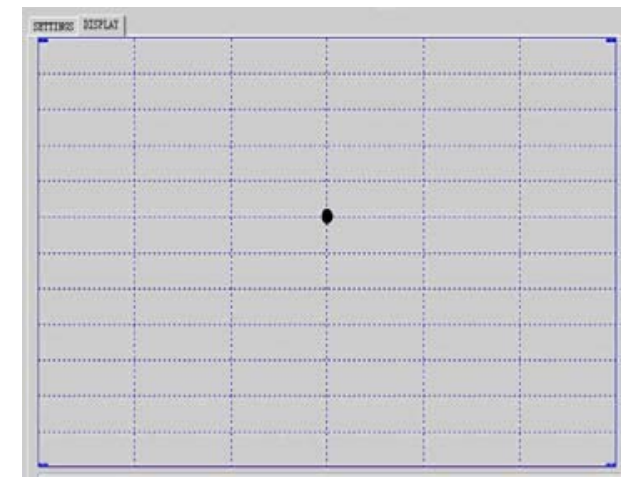

Figure 6. The 2D demo interface of the wireless sensor network localization

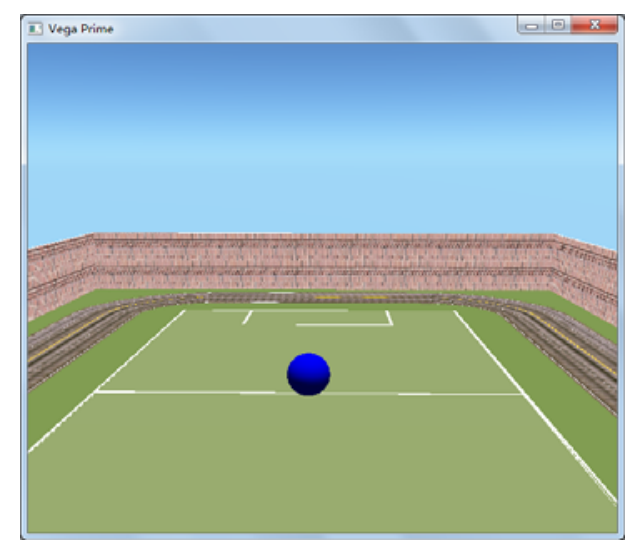

Figure 7. The visual simulation demonstration

\section{CONCLUSION}

Visual simulation is an important part of simulation system. This paper according to simulation application steps based on Vega Prime, designed and realized the visual simulation system of node localization for wireless sensor network in Windows platform. By using Creator modeling and texture processing, then using Vega Prime and VS2005 we did the real-time visual simulation development for node localization in wireless sensor network and had done the vivid demonstration. It solves the problem of general $2 \mathrm{D}$ demo that lacks three-dimensional character and verisimilitude.

This paper will provide the certain reference value to the practical applications of the node localization visual simulation system for wireless sensor network in the future, such as the $3 \mathrm{D}$ demonstration of fire personnel positioning.

\section{REFERENCES}

[1] J.Z. Wu, G. Dang, H.F. Liu, Zh.Q. Cheng, M. Yang. Visual simulation technology and application. Xi'an Electron University of Science and Technology Press. 2001.

[2] Sh. Wang, Y.J. Yan, F.P. Hu. Wireless sensor network theory and application. Beijing University of Aeronautics and Astronautics Press. 2007.

[3] Higbtower.J. and Borriello.G. Location systems for ubiquitous computing. IEEE Computer, 2001, 34(8): 57-66.

[4] Ch. Wang, J. Zhou, L.J. Li. Creator visualization simulation modeling technology. Huazhong University of Science and Technology Press, 2004.

[5] MultiGen-Paradigm Inc. Vega Prime Desktop Tutor (Version 2.0). 2005. 